\title{
Erratum to: Influence of additives on properties of concrete with recycled aggregate and fly ash
}

\section{Matgorzata Wydra ${ }^{1, \mathrm{a}}$}

${ }^{1}$ Faculty of Civil Engineering, Mechanics and Petrochemistry (Płock), Warsaw University of Technology, Pl. Politechniki 1, 00-661 Warszawa, Poland

Original article: MATEC Web of Conferences 196, 04085 (2018), https://doi.org/10.1051/matecconf/201819604085

1. The table 2 should be corrected:

Table 2. Mixture proportions for $1 \mathrm{~m}^{3}$ of concrete [kg]. [16]

\begin{tabular}{|c|c|c|c|c|}
\hline Component/mixture & REC1 & REC2 & REC3 & REC4 \\
\hline CEM III/A 42.5N & 300 & 300 & 300 & 300 \\
\hline Fly ash & 200 & 200 & 200 & 200 \\
\hline Microsilica & 0 & 50 & 0 & 0 \\
\hline Metakaolin & 0 & 0 & 50 & 0 \\
\hline Fluidized fly ash & 0 & 0 & 0 & 50 \\
\hline Natural sand 0-2 & 538 & 505 & 509 & 498 \\
\hline Natural sand 2-4 & 196 & 184 & 185 & 181 \\
\hline RCA 4-8 & 369 & 347 & 349 & 342 \\
\hline RCA 8-16 & 443 & 416 & 419 & 409 \\
\hline SP FK-88 & 8 & 9 & 9 & 9 \\
\hline Water & 172 & 186 & 185 & 183 \\
\hline (W+SP)/(C+P)* $^{*}$ (S) & 0.360 & 0.390 & 0.388 & 0.384 \\
\hline W/C & 0.573 & 0.620 & 0.617 & 0.610 \\
\hline
\end{tabular}

*Previous to mixing RCA has been saturated, the saturation water is NOT incorporated in the ratio.

1. The caption in the 2 chapter should be corrected:

Fig. 1. Sorptivity test [16]

2. The captions in the 3 chapter should be corrected:

Fig. 3. Damaged specimens after 130 cycles [16]

Table 3. Results of compressive strength tests [16]

Table 4. Results of frost resistant tests - relative decrease of compressive strength [16]

Table 5. Results of sorptivity and absorbability tests [16]

3. The following sentence in the 4 chapter should be added

Author wishes to thank Mr. Patryk Tomczak for help in preparing specimens and carrying out tests.

4. Corrected list of references:

${ }^{a}$ Corresponding author: malgorzata.wydra@pw.edu.pl 


\section{References}

1. W. Kubissa, R. Jaskulski and P. Reiterman, J. of Renewable Mater., Volume 5, Supplement 1, January (2017), pp. 53-61(9).

2. W. Kubissa, T. Simon, R. Jaskulski, P. Reiterman, M. Supera, Procedia Eng.,172 (2017) $595-603$.

3. G. Fathifazl, A. Abbas, A. G. Razaqpur, O. B. Isgor, B. Fournier, S. Foo, J. Mater. Civ. Eng,.21 (10) (2009) 601-611.

4. K.-H. Yang, H.-S. Chung, A. F. Ashour, ACI Mater. J.,105 (3).

5. L. Evangelista, J. De Brito, Cem. Concr. Compos., 29 (5) (2007) 397-401.

6. S. C. Paul, G. P. van Zijl, Int. J. Sustainable Constr. Eng. and Technol., 4 (1) (2013) 89-103.

7. I. B. Topcu, S. Sengel, Cem. Concr. Res.,34 (8) (2004) 1307-1312.

8. M. Limbachiya, T. Leelawat, R. Dhir, Mater. Struct.,33 (9) (2000) 574-580.

9. J. Jain, K. P. Verian, J. Olek, N. Whiting, J.Transp. Res. Board,2290 (1) (2012) 44-51.

10. S. Abukersh, C. Fairfield, Constr. Build. Mater.,25 (10) (2011) 4088-4094.

11. K. McNeil, T. H.-K. Kang, Int. J. Concr. Struct. and Mater.,7 (1) (2013) 61-69.

12. P. A. Claisse, J. G. Cabrera, D. N. Hunt, Adv. Cem. Res., 13, No. 4, October, (2001) 165-174.

13. M. Dąbrowski, M. A. Glinicki, Roads and Bridges,12 (2013) 41-55.

14. B. Łaźniewska - Piekarczyk, Cem.Wapno Bet.May-June 3 (2013).

15. J. Babińska, Build. Res. Inst.Q.,4 (160) (2011).

16. P. Tomczak, Eng. Thesis, Warsaw University of Technology (2017). 